\title{
Pediatric nurses' grit and nursing intention during the COVID-19 pandemic: Mediating and moderating effects of mindset and psychological collectivism
}

\author{
Young Soo Chu ${ }^{1}$, Won-Oak $\mathrm{Oh}^{2}$, Il Tae Park ${ }^{3}$, Anna Lee ${ }^{4}$, Myung-Jin Jung ${ }^{5}$ \\ ${ }^{1}$ Graduate Student, College of Nursing, Korea University, Seoul · Chief Nursing Officer, Korea University Medicine, Seoul; ${ }^{2}$ Professor, College of Nursing, Korea \\ University, Seoul; ${ }^{3}$ Assistant Professor, College of Health and Welfare, Woosong University, Daejeon; ${ }^{4}$ Research Professor, College of Nursing, Korea University, \\ Seoul; ${ }^{5}$ Graduate Student, College of Nursing, Korea University, Seoul, Korea
}

Purpose: This study aimed to identify the mediating and moderating effects of mindset and psychological collectivism, respectively, on the relationship between grit and nursing intention for children with emerging infectious diseases, including coronavirus disease 2019 (COVID-19), among pediatric nurses. Methods: We conducted a crosssectional descriptive study with 230 pediatric nurses. Results: Grit directly impacted mindset $(\beta=.27, p<.001)$ and nursing intention $(\beta=.20, p=.001)$. The direct effect of mindset on nursing intension $(\beta=.28, p<.001)$ and the indirect effect of grit on nursing intension via mindset $(\beta=.08, p<.001)$ were significant. Accordingly, mindset mediated the effect of grit on nursing intention. The effects of grit and psychological collectivism ( $\beta$ $=.19, p=.003$ ), respectively, on nursing intention were significant. However, the interaction between grit and psychological collectivism was not significant. Thus, psychological collectivism did not moderate the effect of grit on nursing intention. Conclusion: The findings demonstrate the importance of pediatric nurses' grit and mindset on their intended care for patients in critical situations. Cultivating a gritty culture and developing interventions to enhance nurses' personality traits associated with their performance will be crucial, and such measures are especially salient for pediatric nurses to face the new adjustments required in the era of COVID-19.

Key words: COVID-19; Pediatric nursing; Personality; Caring; Intention

\author{
Corresponding author \\ Won-Oak Oh \\ College of Nursing, Korea University, 145 \\ Anam-ro, Seongbuk-gu, Seoul 02841, \\ Korea \\ TEL: +82-2-3290-4928 \\ FAX: +82-2-927-4676 \\ E-MAIL: wooh@korea.ac.kr
}

Received Aug 26, 2021

Revised Oct 4, 2021

Accepted Oct 15, 2021

\section{INTRODUCTION}

As an emerging infectious disease (EID)-a category that refers to diseases whose incidence has dramatically increased and is likely to increase further in the near future-the coronavirus disease 2019 (COVID-19) pandemic has generated unprecedented health, social, and economic crises that have been devastating for people worldwide. Accordingly, nurses and other healthcare providers have experienced dramatic changes and challenges amid the international turmoil owing to the COVID-19 pandemic [1].

Although pediatric settings might not experience a tremendous increase in patient volume, as has occurred in adult hospitals, the influence of COVID-19 is equally palpable [2]. In addition to the practices that have changed in all healthcare settings (e.g., wearing personal protective equipment [PPE], COVID-19 screenings), additional adjustments are required for pediatric nurses. For example, the facial expressions of pediatric nurses are crucial in communicating with their patients; however, face masks hamper this developmental approach [2]. Nurses also take more time to assess learning needs and provide information regarding COVID-19 to families in a contact-free way (e.g., using iPads) [2], and these adjustments have become the new normal for pediatric nurses in healthcare systems.

Nurses' new roles demand responses to these changes in the healthcare system. The COVID-19 pandemic emergency situation led to recommendations for PPE wear, telemedicine, and social distancing, and pediatric nurses have experienced both professional responsibility and anxiety about transmis- 
sion [3]. They have reported extreme exhaustion owing to long working hours with PPE and face masks, as well as emotional distress including fear of contagion [4]. Considering the critical situations experienced by pediatric nurses on a daily basis, it is important to identify influential factors that could strengthen or worsen pediatric nurses' attitudes towards and performance with COVID-19 patients.

Grit, as a non-cognitive personality trait, refers to one's passion and perseverance over time to achieve goals [5]. Individuals' grit could be evaluated by identifying their interest, obsession, effort, and tenacity in the presence of challenges, using questions such as "I have overcome setbacks to conquer an important challenge" and "my interests change from year to year" [6]. During the COVID-19 pandemic, cultivating grit among pediatric nurses is indispensable to sustain their engagement in critical situations. Nurses with grit are expected to show a higher level of intention to overcome hardships such as caring for challenging patients (e.g., in contexts where there is a risk of being infected by COVID-19). Studies have shown relationships between grit and core variables associated with the nursing workforce. Jeong et al. [7] reported that nurses who had higher levels of grit showed lower turnover intention as well as higher job engagement. Similarly, nurses with grit revealed greater retention intention and job satisfaction [8].

In addition to grit, mindset and psychological collectivism should be discussed as crucial psychological traits that affect nurses' intention and performance in grave situations. Dweck [9] describes mindset as a set of beliefs framing individuals' understanding and response to themselves and the world. There are two types of mindsets: growth (the belief that intelligence is transformational) and fixed (the belief that intelligence is predetermined) [9]. Individuals with a growth mindset believe that success comes from effort, show persistent responses to obstacles, and tend to focus on proficiency in terms of goal orientation [9]. A growth mindset could positively influence how healthcare providers approach obstacles, respond to critical challenges, and pursue their goals [10]. In this regard, a growth mindset is essential to healthcare providers, especially front-line nurses who make critical decisions and provide direct care to people infected with the virus. It is crucial for Korean pediatric nurses to develop their mindset at the present moment, as they face more challenges since COVID-19 vaccinations for children have not started yet in Korea. A prior study found support for a significant relationship between nurses' mindset and nursing intention to care for patients with EIDs [11], as nurses who had higher levels of mindset showed more nursing intention [11].

Psychological collectivism refers to an individual's attitude toward achieving a common goal and belief that it more effective to fulfill the goal as a group and not alone [12].
Highly collectivist people are likely to emphasize the goals and well-being of their in-groups. A study examining 305 Korean workers revealed that participants' psychological collectivism moderated the relationship between grit and organizational citizenship behaviors (e.g., working hard to carry out the tasks of absent workers) [13]. Nurses are members of a hospital organization with various healthcare professionals, whose ability to communicate and collaborate is essential to achieve the common goal of the hospital [14]. The current global challenge posed by COVID-19 calls for more collectivistic effort in hospitals.

The traumatic situation caused by COVID-19 requires pediatric nurses to accomplish tasks beyond their daily performance in caring for pediatric patients and their families. This devastating event places an additional psychological burden on nurses owing to increased work and home demands. An in-depth and comprehensive understanding of psychological traits that impact nurses' work performance would be salient. To date, no study has examined the relationships among grit, mindset, and psychological collectivism of pediatric nurses. This study aimed to 1) identify pediatric nurses' degree of grit, mindset, psychological collectivism, and nursing intention for children with EIDs, 2) assess relationships among grit, mindset, nursing intention, and psychological collectivism in pediatric nurses, 3 ) determine whether mindset mediates the effect of grit on nursing intention, and 4) assess whether psychological collectivism moderates the relationships between grit and nursing intention (Figure 1). We hypothesized that pediatric nurses with higher levels of grit would show higher nursing intention, and that higher levels of a growth mindset and psychological collectivism would mediate and moderate the effects of grit on nursing intention, respectively.

\section{METHODS}

Ethics statement: This study was approved by the Institutional Review Board of Korea University Medicine (No: K2021-0360-001). Informed consent was obtained from the participants.

\section{Design}

A cross-sectional descriptive study design was used in order to investigate hypothesized causal paths by setting 1) a mediating variable (mindset) between an independent variable (grit) and a dependent variable (nursing intention of children with EIDs) and 2) a moderating variable (psychological collectivism) between an independent variable (grit) and a dependent variable (nursing intention for children with EIDs) (Figure 1). 


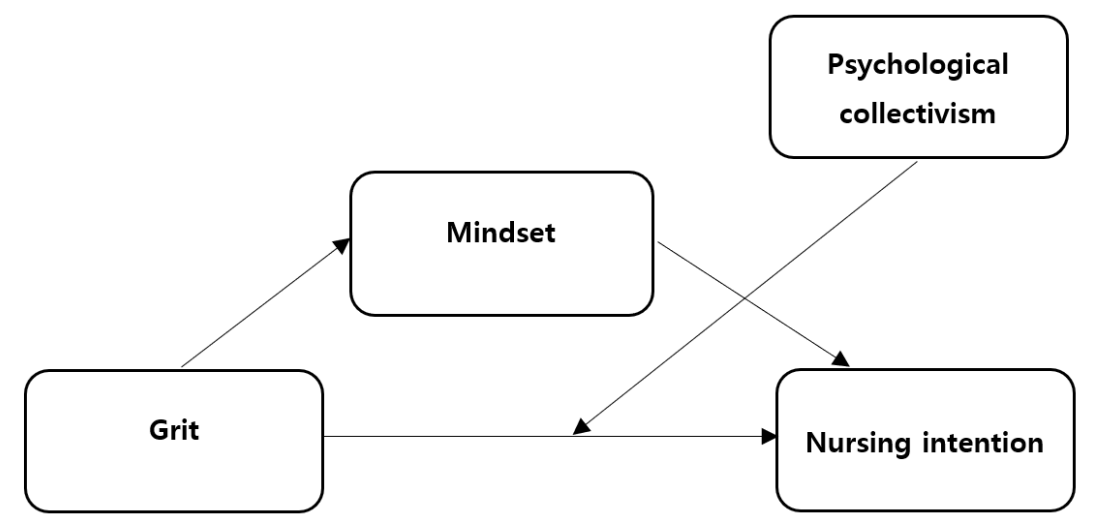

Figure 1. Conceptual relationships among the study variables.

\section{Sample}

The subjects of this study were 240 nurses working in wards for children at Korea University Medicine (two in Seoul and one in Gyeonggi) who understood the purpose of the study and accepted their participation. Studies have found that the first 6 months of new nurses' employment could be the most critical and stressful period for them [15]. Assessments of nurses' psychological traits during this period, therefore, would not reflect their genuine characteristics. Thus, the inclusion criteria were those who had more than 6 months of experience caring for children in a general hospital. After the exclusion of 10 incomplete questionnaires, we finally included 230 subjects in the analysis.

The required sample size was calculated based on the most commonly used bootstrapping technique for determining a mediating effect, which showed that a sample of size at least 200 would be needed for this approach [16]. We assumed that the drop-out rate would be $20 \%$. Furthermore, we collected the questionnaire via postal mail or fax, which could reduce the response rate. However, the response rate was 95.8\%.

\section{Measurements}

1) Grit

Grit was assessed using the Korean version of the Grit Scale [17], which was developed based on the original Grit Scale developed by Duckworth et al. [6]. A total of 12 items were answered on a 5-point Likert scale, with responses ranging from 1 (not at all like me) to 5 (very much like me). This scale includes two dimensions: "perseverance of effort" (6 items) and "consistency of interests" (6 items). Higher scores reflect higher grit (i.e., higher levels of perseverance and passion for long-term goals). The reliability at the time of the tool's development was shown by a Cronbach's $\alpha$ value of .85 [6]. In this study, Cronbach's $\alpha$ was .86 .

\section{2) Mindset}

We measured the mindset of pediatric nurses using the revised Korean version [18] of the original mindset scale [19]. This scale includes two dimensions: growth mindset (five items) and fixed mindset (five items). Each item was rated on a 5-point Likert scale, ranging from 1 (strongly disagree) to 5 (strongly agree). The items for a fixed mindset are reversecoded. Higher scores indicate a higher growth mindset of a pediatric nurse (i.e., the belief that one's intelligence or ability can be changed positively). The reliability at the time of this tool's development was shown by a Cronbach's $\alpha$ value of $.80-.83$ [18]. For the present sample, Cronbach's $\alpha$ was .75.

\section{3) Nursing intention to care for children with emerging in- fectious diseases}

Originally, Yoo et al. [20] developed a nursing intention measurement tool for patients with severe acute respiratory syndrome, and Lee [11] modified the tool for patients with EIDs. Five relevant healthcare experts (three infectious disease nurse practitioners, two medical doctors) reviewed a measurement tool to determine whether it was appropriate to use given the COVID-19 pandemic situation and agreed that it was suitable to use in this study. This tool includes seven dimensions with 46 items: behavioral beliefs, normative beliefs, control beliefs, attitude toward the behavior, subjective norm, perceived behavioral control, and nursing intention. Each item is rated on a 7-point Likert scale, from 1 (do not agree) to 7 (strongly agree). A higher score implies that a pediatric nurse has higher intention to provide nursing care for children with EIDs. The reliability at the time of this tool development was shown by a Cronbach's $\alpha$ of .75-.94 [20]. In this study, Cronbach's $\alpha$ was .83 .

\section{4) Psychological collectivism}

Psychological collectivism was measured using the Korean version of the Psychological Collectivism Scale [21], which 
was translated from the original Psychological Collectivism Scale [12]. This scale includes preference for working in groups (three items), reliance on group members (three items), concern for group members (three items), norm acceptance (three items), and goal priority (three items). Each item is scored on a 7-point Likert scale, with responses ranging from 1 (strongly disagree) to 7 (strongly agree). A higher score indicates a higher degree of psychological collectivism perceived by an individual within the organization. The reliability at the time of this tool development was shown by a Cronbach's $\alpha$ value of .89 [21]. Cronbach's $\alpha$ was .82 in this study.

\section{Study Procedures and Ethical Considerations}

The data collection procedure conformed to the Declaration of Helsinki. After obtaining approval from the university institutional review board of Korea University Medicine (IRB No: K2021-0360-001), data were collected from March to July 2021. With the cooperation of the nursing department of Korea University Medicine, we posted a flyer that recruited participants for this study in a nursing bulletin. Written informed consent was obtained from all participants following an explanation of the study's purpose, methods, risks, and benefits, as well as the rights of the participants. They were informed that they could withdraw from the study at any time. The confidentiality of the obtained information was guaranteed by using code numbers instead of personal identifiers and keeping the paper questionnaires locked in the corresponding author's cabinet. After completing self-reported questionnaires, participants returned them to the research team.

\section{Data Analysis}

We analyzed data using AMOS version 20 and SPSS version 23 (IBM Corp., Armonk, NY, USA). Reliability testing, descriptive statistical analysis, frequency analysis, Duncan post hoc analysis, the t-test, and Pearson correlation analysis were conducted. We employed the Duncan multiple-range test to carry out a post hoc test as a method to reduce the number of pairwise comparisons, since this approach would be more sensitive to detect small mean differences [22]. Path analysis was used to evaluate the fitness of the hypothetical model. The goodness of fit index (GFI), adjusted goodness of fit index (AGFI), root mean square error of approximation, comparative fit index, normed fit index, and standardized root mean residual were used to evaluate the fit of the hypothetical model. A covariance structure analysis was performed using the maximum likelihood method to verify the conformity of the hypothetical path model and to confirm the hypothesis. A bootstrapping method (direct, indirect, and total ef- fects) was used to verify the statistical significance of the mediating effect of the hypothetical model. Statistical significance determined using AMOS was utilized to evaluate the moderating effect.

\section{RESULTS}

\section{Sample Characteristics and Nursing Intention}

A total of 230 nurses participated in this study (Table 1). Their mean age was 29.8 years and there were 221 women (96.1\%). More than half of the participants were general nurses $(n=143,62.2 \%)$ with an average of 6.7 years of nursing experience. They worked in emergency rooms $(n=95,41.3 \%)$, orthopedics units $(n=50,21.8 \%)$, pediatric units $(n=47,20.4 \%)$, and intensive care units $(n=32,13.9 \%)$. Most nurses had achieved a bachelor of nursing degree $(\mathrm{n}=152,66.1 \%)$, were married $(\mathrm{n}=$ $171,74.4 \%)$, and did not have children ( $\mathrm{n}=199,86.5 \%)$.

The majority of nurses $(n=179,77.8 \%)$ had no experience caring for children with EIDs, including COVID-19, while 17 (7.4\%) and 34 (14.8\%) nurses had experience caring for children with confirmed and suspected EIDs, respectively. Additionally, $76.5 \%(\mathrm{n}=176)$ reported that they had received training on wearing PPE and $68.7 \%(n=158)$ reported receiving education on EIDs.

The nursing intention varied according to participants' job position ( $\mathrm{F}=4.09, p=.018)$, whether they had their own children $(\mathrm{F}=4.33, p=.014)$, whether they had experienced education on wearing PPE ( $\mathrm{t}=2.97, p=.003)$ or EIDs $(\mathrm{t}=2.69, p=.008)$ (Table 1).

\section{Grit, Mindset, Nursing Intention, and Psychological Collectivism}

The pediatric nurses had average scores of $(3.10 \pm 0.39)$ and $(3.73 \pm 0.58)$ on the grit and mindset scales, out of 5 points. The mean scores for nursing intention and psychological collectivism were $(3.95 \pm 0.65)$ and $(4.96 \pm 0.81)$ out of a total of 7 points, respectively. All variables had significant relationships with one another (grit and mindset, $\mathrm{r}=.27, p<.001$; grit and nursing intention, $\mathrm{r}=.28, p<.001$; grit and psychological collectivism, $\mathrm{r}=.26, p<.001$; mindset and nursing intention, $\mathrm{r}=.34, p<.001$; mindset and psychological collectivism, $\mathrm{r}=.22$, $p=.001$; nursing intention and psychological collectivism, $\mathrm{r}=.25$, $p<.001)$.

\section{Mediating Effect of Mindset between Grit and Nursing Intention}

The final model yielded a good fit for the data $\left(x^{2}[0]=0.000\right.$, 
Table 1. Sample Characteristics and Differences in Nursing Intention $(N=230)$

\begin{tabular}{|c|c|c|c|c|c|}
\hline \multirow{2}{*}{ Characteristics } & \multirow{2}{*}{ Categories } & \multirow{2}{*}{$\mathrm{n}(\%)$} & \multicolumn{3}{|c|}{ Nursing intention } \\
\hline & & & $\mathrm{M} \pm \mathrm{SD}$ & t or F & $p$ \\
\hline Age (year) & & & $29.8 \pm 5.60$ & & \\
\hline Gender & $\begin{array}{l}\text { Female } \\
\text { Male }\end{array}$ & $\begin{array}{l}221(96.1) \\
9(3.9)\end{array}$ & $\begin{array}{l}3.92 \pm 0.65 \\
4.23 \pm 0.58\end{array}$ & 1.40 & .156 \\
\hline Position & $\begin{array}{l}\text { General nurse }^{\mathrm{a}} \\
\text { Charge nurse }^{\mathrm{b}} \\
\text { Head nurse or manager }^{\mathrm{c}}\end{array}$ & $\begin{array}{c}143(62.2) \\
84(36.5) \\
3(1.3)\end{array}$ & $\begin{array}{l}3.92 \pm 0.67 \\
3.90 \pm 0.58 \\
6.67 \pm 0.58\end{array}$ & 4.09 & $\begin{array}{c}.018 \\
a, b<c^{*}\end{array}$ \\
\hline Career (year) & & & $6.70 \pm 5.60$ & & \\
\hline Department of work & $\begin{array}{l}\text { Pediatric unit } \\
\text { Intensive care unit } \\
\text { Emergency room } \\
\text { Orthopedic unit } \\
\text { Etc. }\end{array}$ & $\begin{array}{c}47(20.4) \\
32(13.9) \\
95(41.3) \\
50(21.8) \\
6(2.6)\end{array}$ & $\begin{array}{l}3.98 \pm 0.63 \\
3.73 \pm 0.54 \\
3.97 \pm 0.69 \\
3.93 \pm 0.65 \\
3.84 \pm 0.70\end{array}$ & 0.96 & .432 \\
\hline Education & $\begin{array}{l}\text { College } \\
\text { RN-BSN } \\
\text { Baccalaureate } \\
\text { Graduate } \\
\text { More than graduate }\end{array}$ & $\begin{array}{c}17(7.4) \\
33(14.3) \\
152(66.1) \\
23(10.0) \\
5(2.2)\end{array}$ & $\begin{array}{l}3.86 \pm 0.94 \\
3.90 \pm 0.59 \\
3.94 \pm 0.64 \\
3.89 \pm 0.59 \\
4.22 \pm 0.60\end{array}$ & 0.35 & .844 \\
\hline Marital status & $\begin{array}{l}\text { Yes } \\
\text { No } \\
\text { Divorce or bereavement }\end{array}$ & $\begin{aligned} & 171(74.4) \\
& 58(25.2) \\
& 1(0.4)\end{aligned}$ & $\begin{array}{l}3.89 \pm 0.61 \\
4.02 \pm 0.75 \\
4.48\end{array}$ & 1.25 & .317 \\
\hline Number of own children & $\begin{array}{l}\text { None }^{a} \\
\text { One } \\
\text { More than } t w o^{c}\end{array}$ & $\begin{array}{c}199(86.5) \\
15(6.5) \\
16(7.0)\end{array}$ & $\begin{array}{l}3.89 \pm 0.61 \\
3.95 \pm 0.71 \\
4.38 \pm 0.94\end{array}$ & 4.33 & $\begin{array}{c}.014 \\
a, b<c^{*}\end{array}$ \\
\hline $\begin{array}{l}\text { Experience of caring for children } \\
\text { with EIDs }\end{array}$ & $\begin{array}{l}\text { No } \\
\text { Confirmed patients } \\
\text { Suspected patients }\end{array}$ & $\begin{array}{l}179(77.8) \\
17(7.4) \\
34(14.8)\end{array}$ & $\begin{array}{l}3.92 \pm 0.66 \\
3.86 \pm 0.83 \\
3.98 \pm 0.50\end{array}$ & 0.20 & .815 \\
\hline $\begin{array}{l}\text { Experience of education on wearing } \\
\text { personal protective equipment }\end{array}$ & $\begin{array}{l}\text { Yes } \\
\text { No }\end{array}$ & $\begin{array}{r}176(76.5) \\
54(23.5)\end{array}$ & $\begin{array}{l}4.00 \pm 0.65 \\
3.70 \pm 0.61\end{array}$ & 2.97 & .003 \\
\hline Experience of education on EIDs & $\begin{array}{l}\text { Yes } \\
\text { No }\end{array}$ & $\begin{array}{r}158(68.7) \\
72(31.3)\end{array}$ & $\begin{array}{l}4.00 \pm 0.62 \\
3.76 \pm 0.68\end{array}$ & 2.69 & .008 \\
\hline
\end{tabular}

*Duncan comparison, $p<.050$; EID, emerging infectious diseases; RN-BSN, registered nurse-bachelor of science in nursing.

$\mathrm{GFI}=849, \mathrm{AGFI}=.698, \mathrm{PGFI}=.425, \mathrm{RMR}=.064)$. The evaluation of the model fit for verifying the mediating effect indicated that the values of $x^{2}$ and degrees of freedom were 0 and GFI=1, which implies that the saturated model always yielded the best GFI. Thus, the observed data were considered to properly reflect the current model [23]. The results showed direct effects of grit on mindset $(\beta=.27, p<.001)$ and nursing intention $(\beta=.20, p=.001)$ (Table 2 and Figure 2$)$. The direct effect of mindset on nursing intention was also significant $(\beta=.28$, $p<.001)$. The indirect effect of grit on nursing intention via mindset was significant $(\beta=.08, p<.001)$. Accordingly, the results supported the hypothesis that mindset was a mediating variable between grit and nursing intention.

\section{Moderating Effect of Psychological Collectivism be- tween Grit and Nursing Intention}

Table 3 and Figure 2 show the results of the path coefficient analysis for moderating effect verification. Grit significantly influenced nursing intention $(\beta=.24, p<.001)$. The effect of psychological collectivism (adjustment variable) on nursing intention (dependent variable) was statistically significant $(\beta=$ $.19, p=.003$ ). However, the interaction between grit and psychological collectivism was not statistically significant $(\beta=.11$, $p=.091)$. Thus, psychological collectivism showed no moderating effect between grit and nursing intention, which did not support the hypothesis that psychological collectivism moderated the effects of grit on nursing intention. 
Table 2. Mediating Effect of Mindset between Grit and Nursing Intention ( $N=230)$

\begin{tabular}{|c|c|c|c|c|c|c|c|c|c|c|c|c|c|}
\hline \multirow{2}{*}{$\begin{array}{c}\text { Independent } \\
\text { variables }\end{array}$} & \multirow{2}{*}{\multicolumn{2}{|c|}{$\begin{array}{c}\text { Dependent } \\
\text { variables }\end{array}$}} & \multirow{2}{*}{$\beta$} & \multirow{2}{*}{ SE } & \multirow{2}{*}{ C.R. } & \multirow{2}{*}{$p$} & \multicolumn{2}{|c|}{ Direct effect } & \multicolumn{2}{|c|}{ Indirect effect } & \multicolumn{2}{|c|}{ Total effect } & \multirow{2}{*}{ SMC } \\
\hline & & & & & & & $\beta$ & $\mathrm{t}(p)$ & $\beta$ & $\mathrm{t}(p)$ & $\beta$ & $\mathrm{t}(p)$ & \\
\hline Grit & $\rightarrow$ & Mindset & .27 & 0.09 & 4.31 & $<.001$ & .27 & $\begin{array}{c}4.31 \\
(<.001)\end{array}$ & & & .27 & $\begin{array}{c}4.31 \\
(<.001)\end{array}$ & 0.08 \\
\hline Grit & $\rightarrow$ & Nursing intention & .20 & 0.11 & 3.23 & .001 & .20 & $\begin{array}{c}3.03 \\
(.003)\end{array}$ & .08 & $\begin{array}{c}3.31 \\
(<.001)\end{array}$ & .28 & $\begin{array}{c}4.18 \\
(<.001)\end{array}$ & 0.15 \\
\hline Mindset & $\rightarrow$ & Nursing intention & .28 & 0.07 & 4.41 & $<.001$ & .28 & $\begin{array}{c}4.41 \\
(<.001)\end{array}$ & & & .28 & $\begin{array}{c}4.41 \\
(<.001)\end{array}$ & \\
\hline
\end{tabular}

$\beta$, standardized estimate; SE, standard error; C.R., critical ratio; SMC, squared multiple correlation.

Table 3. Moderating Effect of Psychological Collectivism between Grit and Nursing Intention

\begin{tabular}{|c|c|c|c|c|c|c|c|}
\hline Independent variables & & Dependent variables & Estimate & $\beta$ & SE & C.R. & $p$ \\
\hline Psychological collectivism & $\rightarrow$ & Nursing intention & 0.15 & .19 & 0.05 & 2.95 & .003 \\
\hline Grit & $\rightarrow$ & Nursing intention & 0.39 & .24 & 0.11 & 3.68 & $<.001$ \\
\hline Grit $\times$ psychological collectivism & $\rightarrow$ & Nursing intention & 0.20 & .11 & 0.12 & 1.69 & .091 \\
\hline
\end{tabular}

$\beta$, standardized estimate; SE, standard error; C.R., critical ratio.

(A)

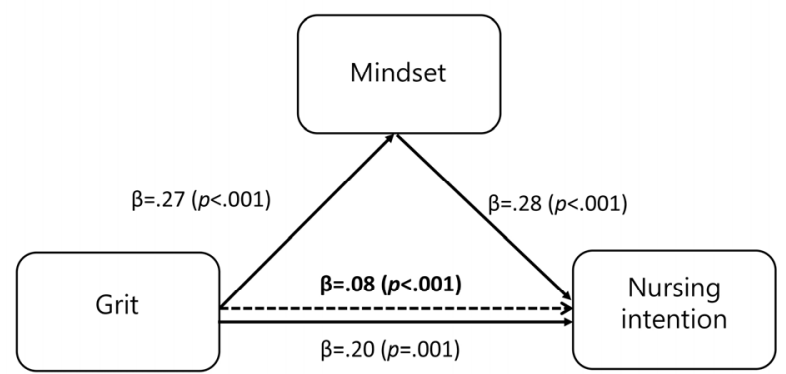

(B)

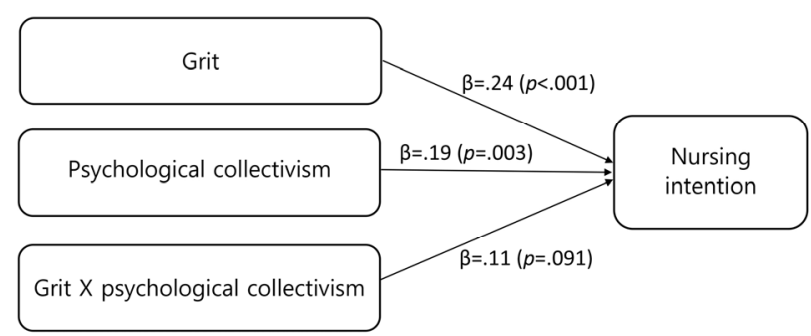

Figure 2. Path model of the study. (A) Mediating effect of mindset between grit and nursing intention. (B) Moderating effect of psychological collectivism between grit and nursing intention.

\section{DISCUSSION}

The challenges of contemporary healthcare systems urgently require additional studies investigating healthcare providers' personality qualities that may be related to work performance, job satisfaction, or longevity in the nursing profession [1]. In particular, the current situation involving immense challenges owing to COVID-19 has intimidated the nursing workforce. To the best of our knowledge, this study is the first attempt to explore the relationships among grit, mindset, psychological collectivism, and the intention to care for children with EIDs including COVID-19 among pediatric nurses.

The results indicate that pediatric nurses with grit showed more intention to care for children with EIDs, corresponding to a greater willingness to care for children with EIDs and positive views on their ability to provide care. The current findings are similar to those reported by Seguin's study [1] that examined 410 nurse leaders. The findings showed that nurses' grit was significantly associated with their increased perceptions of personal accomplishment and reduced burnout [1]. An association between grit and improved performance was established among 669 doctoral students in a variety of programs from business, education, and psychology [24]. Grit compels a person to achieve goals despite difficulties [6]. Nursing children with EIDs could be more challenging physically and emotionally than caring for children with non-EIDs because of the greater fatigue caused by PPEs and fear of contagion [4]. Moreover, pediatric nurses must deal with the family members of 
children with EIDs. The nurses should inform parents about the progression of their children's illness and provide emotional support for families through non-face-to-face methods. Additionally, pediatric nurses have to bear the risk derived from the fact that vaccinations have not been started for Korean children, which could aggravate the fear of contagion [4]. Cultivating grit in pediatric nurses is critical, especially in challenging situations such as the COVID-19 pandemic.

Moreover, the present findings explain the relationship between pediatric nurses' grit and their intention to care for children with EIDs through nurses' mindset. In particular, the results showed that nurses' grit influenced their nursing intention through mindset as a mediating factor. Similarly, Lee and Kim [25] found significant relationships among growth mindset, grit, and self-directed learning ability of 194 nursing students; their results also showed that both growth mindset and grit influenced self-directed learning ability. These findings are consistent with the evidence that grit is a personal trait that can be developed through one's mindset [5]. We particularly focused on a growth mindset, which is characterized as the belief in developing intelligence and the attitude of embracing challenges [9]. It is noted that individuals with grit tend to exhibit an optimistic approach to adversity and believe that their capabilities can be enhanced through effort [5]. Consequently, they spend more time refining and improving their skills. These correspond to the postulates of the growth mindset.

Accordingly, grittier pediatric nurses are expected to have a more optimistic rather than pessimistic attitude towards the hardship caused by COVID-19 and believe that their nursing skills to manage children with COVID-19 can be improved. They are also more likely to dedicate more time to improve their nursing skills in order to improve their nursing practice for children. Enhancing a growth mindset among pediatric nurses is essential for them to make correct decisions and provide accurate nursing services when caring for unvaccinated children. Researchers have advocated for the benefits and importance of growth mindset-oriented education in the health professions [10]. More research on interventions that can enhance mindset and grit among healthcare providers, including pediatric nurses, will be needed.

The current findings, however, do not provide support for the possibility that psychological collectivism moderates the effect of grit on nursing intention. That is, psychological collectivism did not affect the strength and direction of the relationship between grit and nursing intentions. However, this study demonstrated that nurses' psychological collectivism influenced their nursing intention. Studies have addressed the influence of psychological collectivism on nurses' performance; for example, Liou et al. [26] found that nurses' collectivist orientation was significantly associated with their or- ganizational commitment. Researchers have also emphasized that organizational-level actions play a pivotal role in increasing individuals' grit; examples include having common purposes in organizations and promoting a gritty culture through an environment filled with people setting high standards and sharing the same values [27]. Given these findings, it may be plausible that psychological collectivism, which emphasizes organizations' common goals and values rather than those of individuals, would affect the relationships between grit and nursing intentions; however, the study results contradicted this prediction.

Traditionally, Korea has been a strongly collectivistic country [28]. Koreans tend to identify as citizens of their organizations and subordinate their personal goals relative to the group's goals [28]. However, the traditional notions of collectivistic or individualistic cultures, which have been taken for granted across countries, have been revisited. The new generation of Koreans, referred to as the MZ generation (a combination of millennials [Generation $\mathrm{M}$ ] and Generation Z) is often described to have the most individualistic characteristics in Korean history [29]. To an extreme degree, the argument is that the main pillar of Korean culture is no longer collectivism [30]. The mean age of the nurses who participated in this study was 29.8 years, which could be regarded as a young population in Korea. These findings may imply that for contemporary nurses, the intrinsic traits they possess (e.g., grit and mindset) serve as a stronger factor shaping their behavior rather than collectivistic values (e.g., hospitals' organizational goals), particularly in critical situations such as the COVID-19 pandemic.

Contrary to the current study, Lee's study [13] verified the moderating effect of psychological collectivism on the relationship between grit and organizational citizenship behaviors among 305 Korean workers. The inconsistent results regarding the moderating effect of psychological collectivism may have originated from between-subject heterogeneity, including subjects' age, the type of work, length of service, and work positions. Therefore, to gain more in-depth knowledge, additional replication studies using various participants are needed.

Additionally, the results of this study delineate the importance of education regarding new infectious diseases and protective methods. The pediatric nurses who received education on wearing PPE and EIDs exhibited higher levels of nursing intention to care for children with EIDs. Providing comprehensive education on EIDs, including COVID-19, to pediatric nurses could enhance positive perceptions of their competency to care for children with EIDs and their families. The current study also found that nursing intention differed according to some nurses' characteristics, such as their job position and whether they had their own children. Future research as- 
sessing relationships between various characteristics of nurses and nursing intention will be necessary.

Despite the significant findings of this study, it has some limitations. We conducted a cross-sectional survey and recruited pediatric nurses only from a limited range of hospitals. The current study employed a path analysis method. Future studies building and evaluating models are needed to identify more accurate relationships among grit, mindset, psychological collectivism, and nursing intention, such research investigating in which cases psychological collectivism positively or negatively influences nursing intention.

\section{CONCLUSION}

Pediatric nurses need to build their capacity to provide proper nursing care to children and their families regardless of adverse circumstances such as the COVID-19 pandemic. This study provides additional evidence that nurses' personal traits, including grit and mindset, contribute to their intention to care for patients, especially those in critical conditions. Cultivating a gritty culture in clinical settings has become an important virtue for healthcare leaders; an example of a step that could be taken to achieve that goal would be encouraging nurses to work with gritter individuals with high standards and goal achievers. Furthermore, regular mentorship programs with nurses having higher levels of grit or education programs that can enhance nurses' grit could be helpful. Developing interventions to enhance nurses' critical personality traits associated with their performance will also be warranted.

\section{ORCID}

Young Soo Chu

Won-Oak Oh

Il Tae Park

Anna Lee

Myung-Jin Jung

\section{Authors' contribution}

Conceptualization: Young Soo Chu, Won-Oak Oh, Il Tae Park; Data collection: Young Soo Chu, Il Tae Park; Formal analysis: Won-Oak Oh, Il Tae Park, Anna Lee, Myung-Jin Jung; Writingoriginal draft, Writing-review and editing: all authors; Final approval of published version: all authors.

\section{Conflict of interest}

No existing or potential conflict of interest relevant to this article was reported.

\section{Funding}

This study was supported by a 2021 Nursing Research Institute Grant of Korea University.

\section{Data availability}

Please contact the corresponding author for data availability.

\section{Acknowledgements}

This study could not have been undertaken without the support from the pediatric nurses who work at Korea University Medicine. The authors thank them for their contribution to the research.

\section{REFERENCES}

1. Seguin C. A survey of nurse leaders to explore the relationship between grit and measures of success and well-being. Journal of Nursing Administration. 2019;49(3):125-131. https://doi.org/10.1097/NNA.0000000000000725

2. Gralton KS, Korom N, Kavanaugh K, Wenner S, Norr K. COVID19: Impact for pediatric research, evidence-based practice and quality processes and projects. Journal of Pediatric Nursing. 2020;55: 264-265. https://doi.org/10.1016/j.pedn.2020.08.009

3. Coşkun Şimşek D, Günay U. Experiences of nurses who have children when caring for COVID-19 patients. International Nursing Review. 2021;68(2):219-227. https://doi.org/10.1111/inr.12651

4. Arnetz JE, Goetz CM, Arnetz BB, Arble E. Nurse reports of stressful situations during the COVID-19 pandemic: Qualitative analysis of survey responses. International Journal of Environmental Research and Public Health. 2020;17(21):8126.

https://doi.org/10.3390/ijerph17218126

5. Duckworth A. Grit: The power of passion and perseverance. New York, NY: Scibner Book Company; 2016.

6. Duckworth AL, Peterson C, Matthews MD, Kelly DR. Grit: Perseverance and passion for long-term goals. Journal of Personality and Social Psychology. 2007;92(6):1087-1101.

https://doi.org/10.1037/0022-3514.92.6.1087

7. Jeong JY, Seo YS, Choi JH, Kim SH, Lee MS, Hong SH, et al. The influence of grit on turnover intention of university hospital nurses: The mediating effect of job involvement. Journal of Korean Academy of Nursing. 2019;49(2):181-190.

https://doi.org/10.4040/jkan.2019.49.2.181

8. Park MH, Cho SH. Relationships among grit, job satisfaction, and the intention to stay of clinical nurses in Korea. Journal of Korean Clinical Nursing Research. 2019;25(3):285-293.

https://doi.org/10.22650/JKCNR.2019.25.3.285

9. Dweck CS. Mindset: The new psychology of success. New York, 
NY: Random House Digital; 2008.

10. Wolcott MD, McLaughlin JE, Hann A, Miklavec A, Beck Dallaghan GL, Rhoney DH, et al. A review to characterise and map the growth mindset theory in health professions education. Medical Education. 2021;55(4):430-440. https://doi.org/10.1111/medu.14381

11. Lee JY. Factors influencing the intention of nurses to care for emerging infectious diseases patients [master's thesis]. Seoul: Ewha Womans University; 2018. p. 1-115.

12. Jackson CL, Colquitt JA, Wesson MJ, Zapata-Phelan CP. Psychological collectivism: A measurement validation and linkage to group member performance. Journal of Applied Psychology. 2006;91(4): 884-899. https://doi.org/10.1037/0021-9010.91.4.884

13. Lee YJ. Effects of grit on organizational citizenship behavior: A mediation effect of job positive affect and occupational self-efficacy, and a moderation effect of psychological collectivism [master's thesis]. Seoul: Yonsei University; 2018. p. 1-47.

14. Song B, Seomun GA. The influential factors related to organizational citizenship behavior of nurses: With focus on authentic leadership and organizational justice. Journal of Korean Academy of Nursing Administration. 2014;20(2):237-246. https://doi.org/10.11111/jkana.2014.20.2.237

15. Woo MW, Newman SA. The experience of transition from nursing students to newly graduated registered nurses in Singapore. International Journal of Nursing Sciences. 2020;7(1):81-90.

https://doi.org/10.1016/j.ijnss.2019.11.002

16. Woo JP. The concept and understanding of structural equation modeling. Seoul: Hannarae; 2012.

17. Lee S, Sohn YW. What are the strong predictors of academic achievement? -Deliberate practice and grit. Korean Journal of School Psychology. 2013;10(3):349-366. https://doi.org/10.16983/kjsp.2013.10.3.349

18. Kim HS. Relationship between self-differentiation, mindset, and resilience of university students. Asia-pacific Journal of Multimedia Services Convergent with Art, Humanities, and Sociology. 2019;9 (12):1059-1070. https://doi.org/10.35873/ajmahs.2019.9.12.092

19. Dweck CS. Motivational processes affecting learning. American Psychologist. 1986;41(10):1040-1048.

https://doi.org/10.1037/0003-066X.41.10.1040
20. Yoo HR, Kwon BE, Jang YS, Youn HK. Validity and reliability of an instrument for predictive nursing interaction for SARS patient care. Journal of Korean Academy of Nursing. 2005;35(6):1063-1071. https://doi.org/10.4040/jkan.2005.35.6.1063

21. Kwon N, Tak J. The effects of personal characteristics on employee voice behaviors: The moderating effects of organizational trust. Korean Journal of Industrial and Organizational Psychology. 2015; 28(4):565-589. https://doi.org/10.24230/ksiop.28.4.201511.565

22. Kang HC, Han ST, Huh MH. Introduction to SAS ANOVA by learning by example. Paju: Free Academy; 2004.

23. Kang HC. Discussions on the suitable interpretation of model fit indices and the strategies to fit model in structural equation modeling. Journal of The Korean Data Analysis Society. 2013;15(2): 653-668.

24. Cross TM. The gritty: Grit and non-traditional doctoral student success. Journal of Educators Online. 2014;11(3):1-30. https://doi.org/10.9743/JEO.2014.3.4

25. Lee S, Kim J. Growth mindset, grit and self-directed learning ability of nursing students in online education. Journal of the Korean Applied Science and Technology. 2021;38(2):567-578. https://doi.org/10.12925/jkocs.2021.38.2.567

26. Liou SR, Tsai HM, Cheng CY. Acculturation, collectivist orientation and organisational commitment among Asian nurses working in the US healthcare system. Journal of Nursing Management. 2013;21(4):614-623. https://doi.org/10.1111/j.1365-2834.2012.01447.x

27. Tyer-Viola LA. Grit: The essential trait of nurses during a disaster. Journal of Perinatal and Neonatal Nursing. 2019;33(3):201-204. https://doi.org/10.1097/JPN.0000000000000416

28. Kim BS, Atkinson DR, Umemoto D. Asian cultural values and the counseling process: Current knowledge and directions for future research. Counseling Psychologist. 2001;29(4):570-603. https://doi.org/10.1177/0011000001294006

29. Park TW. Generation XYZ with individualities of circles, squares, and triangles. International Journal of Glocal Culture. 2019;8(1):6-9.

30. Yang J, Lie JW. Has the collectivism of Koreans been changed? An exploratory study. Journal of the Korea Contents Association. 2020; 20(12):593-610. https://doi.org/10.5392/JKCA.2020.20.12.593 